\title{
COMMUTATIVE MONOID RINGS AS HILBERT RINGS
}

\author{
ROBERT GILMER
}

\begin{abstract}
Let $S$ be a cancellative monoid with quotient group of torsion-free rank $\alpha$. We show that the monoid ring $R[S]$ is a Hilbert ring if and only if the polynomial ring $R\left[\left\{X_{i}\right\}_{i \in I}\right]$ is a Hilbert ring, where $|I|=\alpha$.
\end{abstract}

Assume that $R$ is a commutative unitary ring and $G$ is an abelian group. The first research problem listed in [K, Chapter 7] is that of determining equivalent conditions in order that the group ring $R[G]$ should be a Hilbert ring. Matsuda has considered this question in $[\mathbf{M 1}, \S 6]$. His results show that for $G$ finitely generated, $R[G]$ and $R$ are simultaneously Hilbert rings; if $G$ is not finitely generated, then $R[G]$ a Hilbert ring implies that $R$ is a Hilbert ring, but the converse fails. In [M2, §5] Matsuda considers briefly the corresponding Hilbert-ring-characterization problem for a monoid ring $R[S]$, where $S$ is torsion-free and cancellative. Let $\alpha$ be the torsion-free rank of $G$ and let $\left\{X_{i}\right\}_{i \in I}$ be a set of indeterminates over $R$ of cardinality $\alpha$. In Corollary 1 we show that $R[G]$ and $R\left[\left\{X_{i}\right\}_{i \in I}\right]$ are simultaneously Hilbert rings. While the problem of determining equivalent conditions for $R\left[\left\{X_{i}\right\}_{i \in I}\right]$ to be a Hilbert ring has not been completely resolved, it has been worked on extensively [ $\mathbf{K r}$, Go, L, G1, H], and a significant body of positive results exists concerning this problem. The proof of Theorem 1 suggests the following conjecture: if $S$ is a cancellative commutative monoid with quotient group $G$, then $R[S]$ is a Hilbert ring if and only if $R[G]$ is a Hilbert ring; this result is established in Theorem 2.

All monoids considered are assumed to be commutative, and rings are assumed to be commutative and unitary. The statement of Theorem 1 uses the following terminology. For a cardinal number $\alpha$, an extension ring $T$ of $R$ is said to be $\alpha$-generated over $R$ if $T$ is generated over $R$ by a set of cardinality at most $\alpha$.

THEOREM 1. Assume that $X=\left\{X_{i}\right\}$ is a set of indeterminates of cardinality $\alpha$ over the ring $R$. Denote by $Z^{\alpha}$ the direct sum of $\alpha$ copies of the additive group $Z$ of integers. The following conditions are equivalent.

(1) $R[X]$ is not a Hilbert ring.

(2) There exists a prime ideal $P$ of $R$ such that $R / P$ admits an $\alpha$-generated extension ring that is a $G$-domain, but not a field.

(3) The group ring $R\left[Z^{\alpha}\right]$ is not a Hilbert ring.

Received by the editors June $18,1984$.

1980 Mathematics Subject Classification. Primary 13B25, 13B99; Secondary 20C07, 20 M25.

Key words and phrases. Monoid ring, group ring, Hilbert ring, $G$-ideal, $G$-domain, pseudoradical.

${ }^{1}$ Research supported by NSF Grant MCS 8122095. 
Proof. (1) $\Rightarrow(2)$. If $R[X]$ is not a Hilbert ring, then there exists a $G$-ideal $Q$ of $R[X]$ that is not maximal [G2, Theorem 31.8]. Hence, if $P=Q \cap R$, then $R[X] / Q$ is an $\alpha$-generated extension ring of $R / P$ that is a $G$-domain, but not a field.

(2) $\Rightarrow$ (3). Let $D=R / P$ and let $J=D\left[\left\{a_{i}\right\}_{i \in I}\right]$ be an $\alpha$-generated extension domain of $D$ that is a $G$-domain, but not a field. With an eye toward proving Theorem 2, we show that if $T$ is a subring of $D\left[\left\{X_{i}\right\},\left\{X_{i}^{-1}\right\}\right] \simeq D\left[Z^{\alpha}\right]$ containing $D[X]$, then $T$ is not a Hilbert ring. Since $J$ is a $G$-domain, it has nonzero pseudoradical [G3, Theorem 3; G2, Theorem 31.1; Ka, §§1-3]. Choose a nonzero element $b$ in the pseudoradical of $J$. Then $1+b a_{i}$ is a unit of $J$ for each $i$, so

$$
D\left[\left\{1+b a_{i}\right\},\left\{\left(1+b a_{i}\right)^{-1}\right\}\right] \subseteq J .
$$

The $D$-homomorphism of $D\left[\left\{X_{i}\right\}\right]$ onto $D\left[\left\{1+b a_{i}\right\}\right]$ determined by $X_{i} \rightarrow 1+b a_{i}$ admits an extension $\sigma$ to a $D$-homomorphism of $D\left[Z^{\alpha}\right]$ onto

$$
D\left[\left\{1+b a_{i}\right\},\left\{\left(1+b a_{i}\right)^{-1}\right\}\right],
$$

and under $\sigma$ we have

$$
J \supseteq \sigma(T) \supseteq D\left[\left\{1+b a_{i}\right\}\right]=D\left[\left\{b a_{i}\right\}\right] .
$$

Now

$$
\sigma(T)\left[b^{-1}\right] \supseteq D\left[\left\{b a_{i}\right\}, b^{-1}\right] \supseteq D\left[\left\{a_{i}\right\}, b^{-1}\right] \supseteq J\left[b^{-1}\right],
$$

the quotient field of $J$. Consequently, $\sigma(T)\left[b^{-1}\right]$ is the quotient field of $J$, and $\sigma(T)$ is a $G$-domain. If $\sigma(T)$ were a field, then $\sigma(T)\left[b^{-1}\right]$, and hence $J$, would be algebraic over $\sigma(T)$, and this contradicts the fact that $J$ is not a field. Therefore $\sigma(T)$, and hence $T$, is not a Hilbert ring. In particular, $D\left[Z^{\alpha}\right] \simeq R\left[Z^{\alpha}\right] / P\left[Z^{\alpha}\right]$ is not a Hilbert ring, and hence neither is $R\left[Z^{\alpha}\right]$.

(3) $\Rightarrow(1)$. If $\alpha$ is finite, then (3) implies that $R$ is not a Hilbert ring, and hence neither is $R[X]$. If $\alpha$ is infinite, then $R\left[Z^{\alpha}\right]$ is $\alpha$-generated over $R$, and thus is a homomorphic image of $R[X]$. Therefore (1) follows in this case as well. This completes the proof of Theorem 1 .

COROLlaRY 1. Assume that $G$ is an abelian group of torsion-free rank $\alpha$. If $\left\{X_{i}\right\}_{i \in I}$ is a set of indeterminates over $R$ of cardinality $\alpha$, then $R[G]$ and $R\left[\left\{X_{i}\right\}_{i \in I}\right]$ are simultaneously Hilbert rings. In particular, if $\alpha$ is finite, then $R[G]$ is a Hilbert ring if and only if $R$ is a Hilbert ring.

Proof. Choose a free subgroup $F$ of $G$ such that $G / F$ is a torsion group. Then $R[G]$ is integral over $R[F]$, and hence $R[G]$ and $R[F]$ are simultaneously Hilbert rings. Since $F \simeq Z^{\alpha}$, the result then follows from Theorem 1 .

If $Z_{0}$ denotes the additive monoid of nonnegative integers, then $R\left[\left\{X_{i}\right\}_{i \in I}\right]$ is isomorphic to the monoid ring of $Z_{0}^{\alpha}$ over $R$, where $\alpha=|I|$. Moreover, $Z^{\alpha}$ is the quotient group of $Z_{0}^{\alpha}$. Does the equivalence of conditions (1) and (3) of Theorem 1 generalize to the case of an arbitrary cancellative monoid and its quotient group? Theorem 2 shows that this question has an affirmative answer. Theorem 1 is used in the proof of Theorem 2 .

THEOREM 2. If $S$ is a cancellative monoid with quotient group $G$, then $R[S]$ and $R[G]$ are simultaneously Hilbert rings. 
Proof. Let $\alpha$ be the torsion-free rank of $G$ (according to the terminology of [G4, p. 165], $\alpha$ is also referred to as the torsion-free rank of $S$ ). If $\alpha$ is finite, then Theorem 2 follows from [G5, Corollary 1]. If $\alpha$ is infinite, then choose a free subset $F$ of $S$ such that $G / H$ is a torsion group, where $H$ is the subgroup of $G$ generated by $F$. Let $T=S \cap H$. We observe that $R[S]$ is integral over $R[T]$. This is true since, for $s \in S$, there exists a positive integer $n$ such that $n s \in H \cap S=T$. Since the extensions $R[H] \subseteq R[G]$ and $R[T] \subseteq R[S]$ are integral, $R[G]$ and $R[H]$ are simultaneously Hilbert rings, and the same is true of $R[T]$ and $R[S]$. This reduces the proof of Theorem 2 to the case where $G=Z^{\alpha}$ and $S$ is a submonoid of $G$ containing $Z_{0}^{\alpha}$.

Suppose $R\left[Z^{\alpha}\right]$ is not a Hilbert ring. There exist a prime ideal $P$ of $R$ and an $\alpha$-generated extension of $R / P$ which is $G$-domain but not a field. The proof that (2) implies (3) in Theorem 1 then shows that no domain between $(R / P)\left[Z_{0}^{\alpha}\right]$ and $(R / P)\left[Z^{\alpha}\right]$ is a Hilbert ring. In particular, $(R / P)[S]$ is not a Hilbert ring, so neither is $R[S]$. Conversely, if $R[S]$ fails to be a Hilbert ring then, since $\alpha$ is infinite, $|S|=\alpha$ and $R[S]$ is a homomorphic image of $R\left[Z_{0}^{\alpha}\right]$; therefore neither $R\left[Z_{0}^{\alpha}\right]$ nor $R\left[Z^{\alpha}\right]$ is a Hilbert ring in this case.

We referred in the introduction to work that has been done on the problem of determining conditions under which a polynomial ring in infinitely many indeterminates over a Hilbert ring is again a Hilbert ring. To illustrate the relation between some of this work and Theorems 1 and 2, we record a result labelled as Theorem 3. Part (a) of this result, a restatement of [G5, Corollary 1], follows from Theorems 1 and 2; part (b) uses the same two theorems and Theorem 2.9 of [G1], while part (c), which generalizes (b), is a consequence of Theorems 1 and 2 and [H, Theorem 1].

THEOREM 3. Assume that $R$ is a commutative unitary ring and that $S$ is a cancellative monoid of torsion-free rank $\alpha$.

(a) If $\alpha$ is finite, then $R[S]$ is a Hilbert ring if and only if $R$ is a Hilbert ring.

Suppose that $\alpha$ is infinite.

(b) If $R$ is a field, then $R[S]$ is a Hilbert ring if and only if $\alpha<|R|$.

(c) If $R$ is a Noetherian ring (or, more generally, if $\operatorname{Spec}(R)$ is Noetherian and $R$ satisfies d.c.c. on prime ideals), then $R[S]$ is a Hilbert ring if and only if the following conditions are satisfied.

(i) $|R / M|>\alpha$ for each maximal ideal $M$ of $R$.

(ii) For each nonmaximal prime $P$ of $R$, the set of primes $Q$ of $R$, such that $Q>P$ and $\operatorname{ht}(Q / P)=1$, has cardinality greater than $\alpha$.

\section{REFERENCES}

[G1] R. Gilmer, On polynomial rings over a Hilbert ring, Michigan Math. J. 18 (1971), 205-212.

[G2] _ Multiplicative ideal theory, Dekker, New York, 1972.

[G3] _ The pseudo-radical of a commutative ring, Pacific J. Math. 19 (1966), 275-284.

[G4] ___ Commutative semigroup rings, Univ. of Chicago Press, Chicago, 1984.

[G5] __ Hilbert subalgebras generated by monomials, Comm. Algebra (to appear).

[Go] O. Goldman, Hilbert rings and the Hilbert Nullstellensatz, Math. Z. 54 (1951), 136-140.

[H] W. Heinzer, Polynomial rings over a Hilbert ring, Michigan Math. J. 31 (1984), 83-88. 
[K] G. Karpilovsky, Commutative group algebras, Dekker, New York, 1983.

[Ka] I. Kaplansky, Commutative rings, Allyn \& Bacon, Boston, Mass., 1970.

[Kr] W. Krull, Jacobsonsche Ringe, Hilbertscher Nullstellensatz, Dimensionstheorie, Math. Z. 54 (1951), 354-387.

[L] S. Lang. Hilbert's Nullstellensatz in infinite-dimensional space, Proc. Amer. Math. Soc. 3 (1952), 407-410.

[M1] R. Matsuda, Torsion-free abelian group rings. III, Bull. Fac. Sci. Ibaraki Univ. Ser. A 9 (1977), $1-49$.

[M2] Torsion-free abelian semigroup rings. IV, Bull. Fac. Sci. Ibaraki Univ. Ser. A 10 (1978), $1-27$.

Department of Mathematics, Florida State University, Tallahassee, Florida 32306 